\title{
Teoria do Caos Aplicada ao Gerenciamento dos Riscos de um Projeto: Uma Análise Explicativa Sobre o Conceito de Caos nos Processos Sensitivos aos Riscos em um Projeto
}

\author{
QUEIROZ, Diogo Barbosa de ${ }^{[1]}$
}

QUEIROZ, Diogo Barbosa de. Teoria do Caos Aplicada ao Gerenciamento dos Riscos de um Projeto: Uma Análise Explicativa Sobre o Conceito de Caos nos Processos Sensitivos aos Riscos em um Projeto. Revista Científica Multidisciplinar Núcleo do Conhecimento. Ano 03, Ed. 05, Vol. 06, pp. 111-126, Maio de 2018. ISSN:2448-0959

\section{Resumo}

Este trabalho visa explicar de forma objetiva, a teoria do caos e seus conceitos, a influência dessa teoria no gerenciamento dos riscos de um projeto e porque gerenciar os riscos de forma competente pode diminuir a ocorrência de um evento caótico que atrapalhe o desenvolvimento do determinado projeto. A teoria do caos pode ser considera uma teoria recente e inovadora, com aplicações em diversas áreas de estudo. Em projetos ela explica que pequenas condições inicias, podem ocasionar grandes impactos posteriores no projeto ou no produto final. De forma conceitual e aplicada, essa teoria modificará a maneira de pensar em eventos do cotidiano. As aplicações desse estudo são diversas, as primeiras equações foram aplicadas para descrever fenômenos meteorológicos, variação de previsões nos mercados financeiros, movimento de placas tectônicas etc., agora vamos ver a aplicação da teoria do caos junto aos processos de gerenciamento dos riscos de projetos e entender como pequenos eventos isolados no curto prazo podem influenciar um evento maior ao longo prazo, evento que pode ser da mesma natureza ou não. O presente artigo se caracteriza como analise explicativa descritiva, feita através de pesquisas bibliográficas, e como literatura principal sobre gerenciamento de riscos, o guia do conhecimento e gerenciamento de projetos PMBOK, quinta edição.

Palavras chave: Teoria do Caos, Gerenciamento de Riscos, PMBOK.

\section{Introdução}

Com base no gerenciamento de riscos e nos conceitos de caos, apresenta-se aqui como a teoria do caos se aplica positivamente ou negativamente no desenvolvimento de um projeto, durante as fases de 
gerenciamento dos riscos do mesmo. Alguns projetos são muito complexos, como a construção de um estádio, o desenvolvimento de um software, reformulação de uma estrutura organizacional. A teoria do caos explica que, pequenos fatores e eventos que ocorrem de maneira instável ou imperceptível no presente, podem no futuro em um grande lapso temporal mudar completamente o que foi planejado ou programado no inicio de um projeto, ou seja, essa ciência apresenta grande imprevisibilidade e sensibilidade que qualquer sistema dinâmico possui LORENZ (1996). Neste sentido, é proposto que, nos processos de avaliação dos riscos do projeto como um todo, atrelado aos objetivos e fases do projeto, tais como escopo, custo, tempo e qualidade, como pequenos erros em diferentes etapas podem causar variações nessas quatro variáveis que formam o "triângulo de ferro" do projeto, e com isso mudar completamente o que foi planejado e assim interferir na qualidade do produto ou serviço final (PMBOK, 2013).

Ao final desta leitura, será notória uma reavaliação sobre os conceitos ambientais que influenciam o sucesso de um projeto, não apenas no gerenciamento dos riscos do mesmo. Por definição do PMBOK (2013, p. 03), projeto é:

"Projeto é um esforço temporário empreendido para criar um produto, serviço ou resultado exclusivo. A natureza temporária dos projetos indica que eles têm um início e um término definidos. $\mathrm{O}$ término é alcançado quando os objetivos do projeto são atingidos ou quando o projeto é encerrado porque os seus objetivos não serão ou não podem ser alcançados, ou quando a necessidade do projeto deixar de existir."

Percebe-se que a citação acima, define que o término do projeto pode ser ocasionado pelo não cumprimento dos objetivos planejados. A verdade é que, mesmo o gerenciamento de todas as áreas de conhecimentos, estabelecido pelas boas práticas sejam feitas de maneira competente, oque realmente acontece é imprevisível.

O estudo do caos e sua teoria explicam as variações em condições iniciais em algum evento, ou em algum ponto dinâmico, pode propagar situações inimagináveis, (LORENZ, 1996). O Mundo globalizado, como nós o conhecemos, está em constante mudança, o mesmo é muito sensível a condições econômicas, meteorológicas, humanitárias. Hoje é muito difícil de fazer previsões seguras, mesmo sabendo ou prevenindo riscos em relação aos temas já citados anteriormente (BARALDINE, 2013). No gerenciamento de projetos o caos pode ser teorizado como uma má gestão durante seu planejamento estratégico, durante a iniciação do projeto, na formação da equipe, por conta do ambiente organizacional, por conta do momento errado, ou na fase de execução, este ultimo menos sensitivo as variações, pois os riscos tem um grau maior de probabilidade de ocorrência nas fases iniciais do projeto. É claro que o caos nem sempre é uma coisa ruim. No sentido de pura desordem, realmente, no gerenciamento de um projeto pouco se pode dizer a seu favor (PMBOK, 2013).

Segundo o Guia PMBOK (2013), gerenciar os riscos do projeto inclui os processos envolvidos em estimativas, planejamento, analise quantitativa, qualitativa e controle dos riscos, de modo que o projeto possa ser terminado dentro do orçamento aprovado, do escopo aplicado e do tempo estimado, caso isso ocorra, pode-se dizer que o produto ou serviço final, foi entregue com qualidade, satisfazendo as partes interessadas. A origem do risco é diversa, e muda variavelmente o ambiente que deu origem a metodologia do gerenciamento dos riscos. O risco pode ser causado por diversos fatores. Risco e caos são palavras que assustam qualquer organização. 


\section{Teoria do caos}

Os primeiros estudos dessa teoria surgiram nos anos 60, pelo professor do MIT (Instituto de Tecnologia de Massachusetts), Edward Norton Lorenz (1817- 2008), que foi o precursor desse estudo. O conceito do caos e sua teoria contradizem a ciência clássica que apresenta formulas lógicas que explicam e preveem a ordem de eventos que acontecem no mundo em sistemas lineares quaisquer. Portanto, espera-se que as causas essenciais de ocorrência desses eventos sejam conhecidas. No entanto, a teoria do caos usa conceitos e equações não lineares, onde uma pequena e a principio insignificante alteração nas condições inicias de um sistema, leve ao final um estado completamente diferente daquele previsto no inicio.

Para Lorenz (1996, p. 195), a definição de um processo linear é:

"aquele no qual uma mudança em qualquer variável em algum instante inicial produzir uma mudança nela mesma ou em outra variável em algum instante posterior, uma mudança duas vezes maior, no mesmo instante inicial, irá produzir uma mudança duas vezes maior no mesmo instante posterior. Você pode substituir duas vezes por metade ou por cinco vezes ou por cem vezes e a definição continuará válida”.

O conceito de Caos aponta uma desorganização, uma desordem na qual as incertezas e imprevisibilidades são predominantes, porém pode-se incluir a teoria do caos em conceitos da natureza, levando em consideração as noções de probabilidade (podendo ou não ocorrer), aleatoriamente (ocorrência propagada ao acaso), sensibilidade (pequeno desvio pode ser amplificado exponencialmente) e irreversibilidade (uma vez ocorrido, torna-se improvável o seu retorno no estado inicial) (PRIGOGINE, 2002).

Então, Caos é o comportamento aperiódico de longo prazo em um sistema determinístico que exibe sensibilidade às condições iniciais. Então, qualquer sistema que não se comporta de forma linear, pode ser definido como um sistema caótico. A teoria do caos, às vezes é expressa de forma dramática, quando se apresenta o efeito borboleta de LORENZ (1963). "O bater de asas de uma borboleta em Nova York, pode fazer chover em Tóquio."

\section{Gerenciamento de riscos}

No Guia PMBOK (2013) são descritas 10 áreas do conhecimento que abrangem todo o ciclo de um projeto, desde o seu planejamento, passando por sua execução até o seu encerramento. Durante essas fases, a diversas possibilidades de erros que podem ocorrer. Aquela frase famosa e dita muitas vezes no meio acadêmico "na teoria é uma coisa, na prática é outra" cai muito bem com esse estudo, ao passo que a muitas variáveis existentes em um processo de gestão de riscos, ou quaisquer processos de um projeto, e muitas dessas variáveis são planejadas, ou qualificadas e muito menos quantificadas. O não gerenciamento de riscos ou o gerenciamento inadequado, pode transformar o desenvolvimento do projeto um caos., ao passo que outros erros possam ocorrer como: muitas alterações de escopo, prazos arrojados, falta de comunicação..., podem ocorrer por incompetência no planejamento dos processos do projeto. Esses problemas aumentam o risco de algum evento negativo vir a ocorrer. Portanto saber gerenciar os riscos é um trabalho fundamental que deve ser muito bem executado.

Gerenciamento de Riscos é o processo de planejar, organizar, dirigir e controlar os recursos humanos e materiais de uma organização, de um projeto, ou de um empreendimento, no sentido de minimizar os efeitos dos riscos negativos sobre essa organização ao mínimo possível. É um conjunto de técnicas que 
visa reduzir ao mínimo os efeitos das perdas acidentais, enfocando o tratamento aos riscos que possam causar danos ao contexto em que esta sendo aplicado (HUBBARD, 2007).

O gerenciamento de riscos é de fundamental importância para a sobrevivência e harmonização de qualquer sistema dinâmico aplicado, razão pela qual se tornou um das principais preocupações das organizações mundiais. A noção do risco pode ser classificada e diversificada, a partir da metodologia de aplicação. (BARALDINE, 2013).

O risco de um projeto é uma condição ou evento incerto que se ocorrer, desencadeará um efeito negativo ou positivo em um ou mais objetivos do projeto. Os riscos do projeto tem Origem nas incertezas que circundam todo o gerenciamento do projeto, como qualidade, escopo, tempo e custo. (PMBOK, 2013).

Abaixo, segue como exemplo, uma pesquisa realizada no Brasil, sobre as principais causas de fracassos em projetos em empresas do ramo de tecnologia da informação:

CAUSAS DE FRACASSO

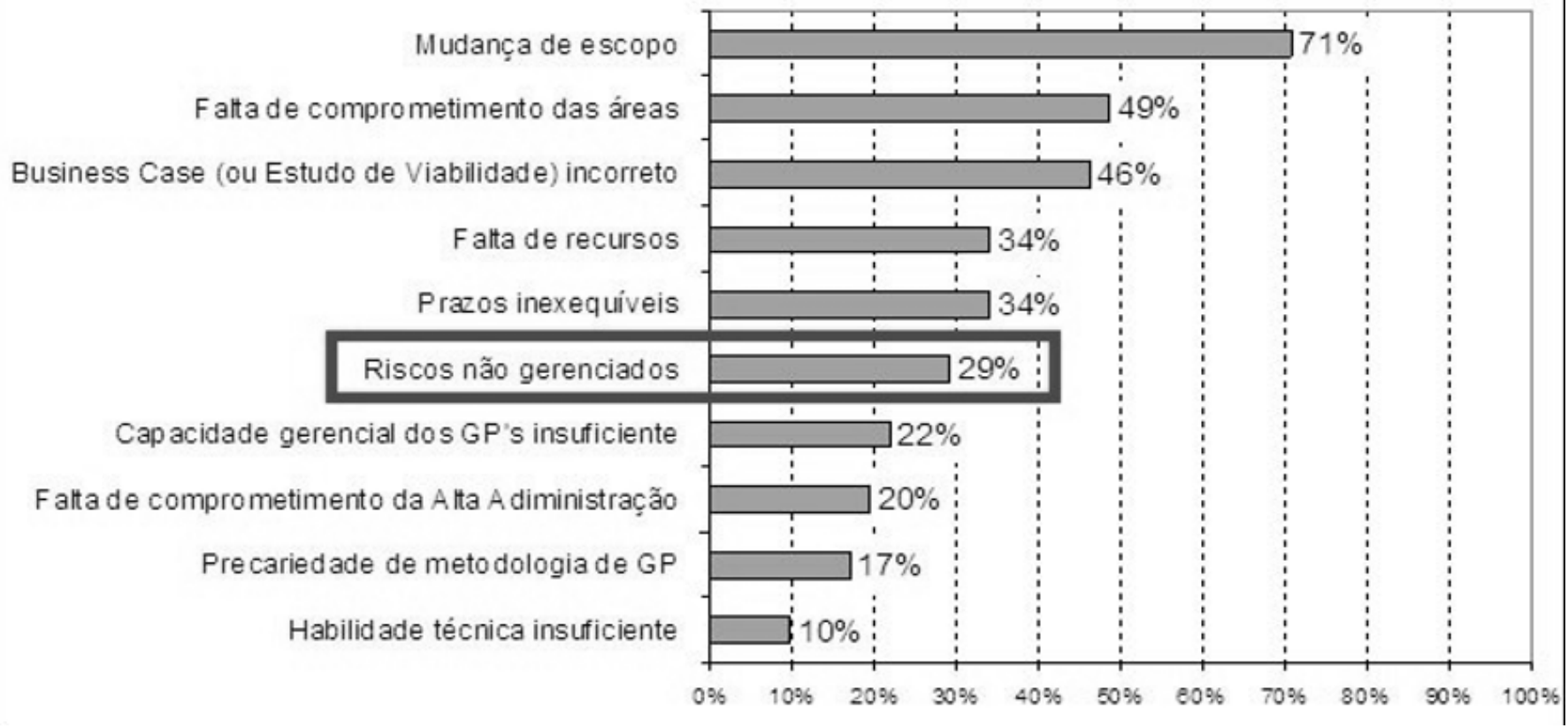

Figura 1- Gráfico de barras representando as principais causas de fracassos em empresas. Fonte: Análise dos resultados. Archibald \& Prado (t.i.), 2011.

Cerca de $29 \%$ das causas de fracassos em projetos são devidos ao não gerenciamento dos riscos. Nota-se que apesar da pesquisa quantificar outros temas como mais relevantes de causas de fracassos, o gerenciamento dos riscos aparece com porcentagem significativa.

\section{Teoria do caos aplica no gerenciamento dos riscos no projeto}

De acordo com o Guia PMBOK (2013, p. 238), “o risco do projeto é um evento ou condição incerta que, se ocorrer, terá um efeito positivo ou negativo sobre pelo menos um objetivo do projeto, como tempo, custo, escopo ou qualidade". Os riscos do projeto estão atrelados às incertezas que cada projeto possui, as 
empresas entendem assim. Os gerentes e partes interessadas devem avaliar a eminencia ou não de determinados risco, e saber gerencia-los, ao passo que existem riscos já sabidos e riscos imprevisíveis, atrelados a fatores externos como, mercado financeiro, condições ambientais, fator humano... (BARALDI, 2013).

O estudo do caos apresenta trabalhos aplicados em todas as áreas de conhecimento, como; engenharia, biologia, meteorologia, sociologia, política e etc. Aplicadas no mundo o qual o conhecemos e suas mudanças constantes e imprevisíveis. Com base nos estudos de GATTI (2007), os modelos lineares, estatísticos e padrões determinísticos, são postos como alternativas limitadas, pois uma simples variável em algum cálculo determinístico, ou alguma mudança imperceptível no ambiente organizacional de um projeto, pode alterar ao longo e/ou curto prazo, todo o planejamento feito, a fim de não garantir que o projeto seja executado no prazo estimado, no escopo aplicado e no custo orçado, e com as incertezas mínimas relacionadas aos pilares do projeto (escopo, custo e tempo), este ultimo talvez o mais sensível a teoria do caos, pois a história desse estudo já apresentou muitos trabalhos aplicados, aos riscos e incertezas no gerenciamento de projetos KERZNER (2009), e a história nos ensina que os riscos podem ser mínimos, caso o gerenciamento dos mesmos seja feito com qualidade, competência e atenção, a fim de se evitar o caos nas fases posteriores do projeto.

A princípio, as etapas das áreas de conhecimento do guia de boas práticas de gerenciamento de projetos, foram vistas como processos lineares e não dinâmicos. Mas ao se gerenciar riscos de forma tradicional, os riscos não identificados por falta de visão macro entre os pilares do projeto (escopo, tempo, custo), permaneceram não identificados e não analisados e respondidos, podendo no futuro desmantelar completamente o projeto. Portando a fase de gerenciamento de riscos envolve muitas variáveis que podem se comportar de forma exponencial, dinâmica e não linear. Por exemplo: ao adequar o escopo com os requisitos exigidos e as disponibilidades de execução dos mesmos, entende-se que durante o planejamento dos riscos de em relação ao escopo do projeto, o mesmo sofrer alguma alteração, isso implicara em um reajuste de tempo e custo do projeto. Com isso, novos eventos se desencadearam e o projeto poderá seguir um caminho a qual não foi planejado, podendo se tornar um risco negativo ou uma oportunidade para as outras áreas de conhecimento do projeto. De qualquer forma, não seria responsável pensar nesses processos de forma linear e previsível.

A teoria do caos se relaciona com os processos do ciclo de vida do projeto, ao entendermos que um projeto, depende de tantos esforços e atividades para ser desempenhados, muitas análises e projeções de planejamentos a serem tomadas, que o mesmo acaba sensitivo ao caos em eventos decorrentes das etapas de desenvolvimento do projeto. As incertezas em cada parâmetro estipulado, também estão sensitivas a imprevisibilidade de ocorrência de algum evento caótico, que poderá ou não prejudicar o andamento do projeto. Quando escolhemos o gerenciamento de riscos do projeto, estamos falando de uma etapa fundamental ao analisarmos todas essas incertezas e imprevisibilidades de analises, eventos, cronogramas, fatores humanos, fatores econômicos e ambientas fatores políticos que estão presentes no desenvolvimento de um projeto.

Durante o ciclo de vida de um projeto, podemos, por definição dizer que um projeto seguindo as boas pratica de gerenciamento, deve passar por cinco áreas de conhecimento (iniciação, planejamento, execução, monitoramento e controle e encerramento), em fases de gerenciamento de sessenta e nove processos, o gerencialmente dos riscos estão atrelados a todas essas etapas, seus estudos e avaliações de ocorrência e impacto, estão sendo aplicados durante cada tomada de decisão, cada avanço no cronograma, 
a cada capital aplicado a cada entrada e saída de pessoal, e por ai vai. Segundo o guia PMBOK (2013) os processos de gerenciamento dos riscos estão divididos em:

\subsection{Planejar o gerenciamento dos riscos}

Em qualquer tipo de planejamento, uma coisa é notória, deve-se sempre planejar os objetivos e suas respectivas atividades, a fim de ter um referencial futuro, para atingir o que foi determinado no passado. Isso não é diferente no gerenciamento dos riscos, estimar custos, é poder assumir riscos, escolher patrocinadores, é assumir riscos, gerenciar o tempo, também é assumir riscos. O planejamento dos riscos pode ser muito sensível ao conceito do caos, portanto é suma importância, que durante esse processo as estimativas, as atividades sejam muito bem planejadas, a fim de se diminuir incertezas inicias, para evitar que se transforme em algum processo caótico que leve o projeto a rumos negativos não planejados (KERZNER, 2009).

\subsection{Identificar os riscos}

Nesta etapa, agrega-se muito conhecimento em relação a tudo que se envolvem no projeto, todos os fatores, e atividades a serem empregadas. Os riscos já conhecidos são gerenciados pela equipe, que devem preparar reservas de contingencia, assim que o determinado evento negativo acontecer. Para prevenir eventos futuros, com alta probabilidade de ocorrência de algo que não foi planejado, a equipe de ter uma reserva de gerenciamento, para evitar um caos maior, que atrapalhe a continuidade do projeto. Membros da equipe do projeto, a equipe de gerenciamento dos riscos (se for designada), clientes, especialistas nos assuntos externos à equipe do projeto, usuários finais, outros gerentes de projetos, partes interessadas e especialistas em gerenciamento dos riscos. Embora essas pessoas sejam as principais participantes na identificação dos riscos, todo o pessoal do projeto deve ser encorajado a identificar riscos (PMBOK, 2013). Os riscos identificados devem ser compreendidos e especificados, a fim de poder responder esses eventos de forma eficaz e consistente, para garantir a continuidade do projeto e diminuir a incerteza do caos existente no presente e no futuro das fases do projeto. Métodos como, diagrama de causa e efeito, diagrama de Pareto, Matriz SWOT e fluxogramas, são exemplos de ferramentas utilizadas nesse processo.

É importante citar os conceitos dessas ferramentas da qualidade:

1. O Diagrama de Causa e Efeito proposto originalmente por Kaoru Ishikawa na década de 60, fez com que as pessoas pensassem sobre causas e razões raízes possíveis que fazem com que um problema ocorra. É uma ferramenta prática, muito utilizada para realizar análise de causa em avaliação de não conformidades.

2. Para AVELAR (2008), o Diagrama de Pareto é um recurso gráfico utilizado para estabelecer uma ordenação nas causas de perdas que devem ser sanadas. Essa ferramenta é formada por barras verticais tendo como objetivo determinar quais problemas resolver primeiro de acordo com seu valor de escala.

3. O objetivo da Matriz SWOT é definir estratégias para manter pontos fortes (riscos positivos), reduzir a intensidade de pontos fracos (riscos negativos), aproveitando oportunidades e protegendo-se de ameaças (eventos caóticos).

4. Os fluxogramas mostram como as várias etapas de um processo/sistema se inter-relacionam. Muitas vezes feito através de gráficos que ilustram de forma descomplicada a transição de 
informações entre os elementos que o compõem.

\subsection{Realizar a análise qualitativa dos riscos}

A etapa de realizar a análise qualitativa dos riscos é muito sensível as condições adversas que podem ocorrer durante outras etapas do gerenciamento do projeto, ao passo que quando não podemos quantificar algo, o mesmo se torna subjetivo, sensitivo, com métodos ou interpretações diversas.

Nessa etapa, segundo o Guia PMBOK (2013) é feita uma avaliação da prioridade dos riscos identificados na etapa anterior, usando sua probabilidade relativa, ou possibilidade de ocorrência, o impacto de cada risco, o intervalo de resposta caso acorra, a aceitação ou tolerância associada a organização e suas restrições em relação ao tempo, custo e escopo do projeto. A avaliação deve ser feita de forma eficaz, não deixando parcialidade em relação a resposta da equipe do projeto, caso aconteça, elas devem ser corrigidas imediatamente para evitar a influência do caos em etapas futuras do projeto. Essa analise qualitativa dos riscos deve ser realizada durante todo o clico de vida do projeto, ao passo que, como já mencionado, novos riscos podem ser identificados conforme o projeto for passando de fase, ou por causa de algum risco identificado antes com uma probabilidade de ocorrência baixo, mas com um impacto caótico (caso ocorra), venha a acontecer, e imagine que para reparar o dano causado a tal evento, a equipe precisou utilizar uma reserva gerencial planejada antes, mas caso as respostas e reservas financeiras não resolvam o problema do evento negativo causado ao projeto, isso irá abalar o projeto como um todo. Por isso nenhum especificação, ou detalhamento deve deixar de ser analisado nesse processo.

Os riscos podem causar efeitos positivos, transformando em oportunidades para melhoras ou diminuir a incerteza de possíveis eventos negativos do projeto. Para isso é feita a "Avaliação de probabilidade e impacto dos riscos", e uma ferramenta muito utilizada é a Matriz de probabilidade e impacto dos riscos, tem como principal objetivo priorizar o tratamento dos riscos: segue exemplo abaixo: 
Probability and Impact Matrix

\begin{tabular}{|c|c|c|c|c|c|c|c|c|c|c|}
\hline Probability & \multicolumn{5}{|c|}{ Threats } & \multicolumn{5}{c|}{ Opportunities } \\
\hline $\mathbf{0 . 9 0}$ & 0.05 & 0.09 & 0.18 & 0.36 & 0.72 & 0.72 & 0.36 & 0.18 & 0.09 & 0.05 \\
\hline $\mathbf{0 . 7 0}$ & 0.04 & 0.07 & 0.14 & 0.28 & 0.56 & 0.56 & 0.28 & 0.14 & 0.07 & 0.04 \\
\hline $\mathbf{0 . 5 0}$ & 0.03 & 0.05 & 0.10 & 0.20 & 0.40 & 0.40 & 0.20 & 0.10 & 0.05 & 0.03 \\
\hline $\mathbf{0 . 3 0}$ & 0.02 & 0.03 & 0.06 & 0.12 & 0.24 & 0.24 & 0.12 & 0.06 & 0.03 & 0.02 \\
\hline $\mathbf{0 . 1 0}$ & 0.01 & 0.01 & 0.02 & 0.04 & 0.08 & 0.08 & 0.04 & 0.02 & 0.01 & 0.01 \\
\hline & 0.05 & 0.10 & 0.20 & 0.40 & 0.80 & 0.80 & 0.40 & 0.20 & 0.10 & 0.05 \\
\hline
\end{tabular}

Figura 2 - Matriz de probabilidade impacto. A área cinza escuro representa alto risco, a área cinza médio representa baixo risco, a área cinza claro representa riscos moderados.Fonte: (PMBOK, 2013, p. 330).

\subsection{Realizar a análise quantitativa dos riscos}

Nesta etapa é feita uma analise quantitativa dos riscos identificados, transformando-os em informações e dados estatísticos importantes que ajudaram a equipe responsável a analisar os efeitos dos riscos, para respaldar as tomadas de decisões, a fim de evitar o caos, reduzindo as incertezas dos processos do projeto.

Segundo o Guia PMBOK (2013) este processo de analise quantitativa dos riscos, é executado, a partir dos resultados encontrados na etapa anterior (análise qualitativa dos riscos), tendo a associação do caos e impactos significativos no decorrer das etapas do projeto. O processo analisa o efeito agregado de todos os riscos que podem afetar eventos e etapas do projeto, principalmente aqueles etapas mais vulneráveis a um possível caos futuro devido a elementos incertos que compõe determinado evento ou etapa do projeto.

Um método utilizado para fazer análise e estimar eventos, são as distribuições de probabilidades. Muito usada para fazer simulação de etapas do projeto, representam a incerteza em unidades ou valores dos pilares da qualidade do projeto, como o custo, tempo e escopo, são usados para testar eventos incertos, analisados em cenários anteriores, e como tal evento poderá se comportar caso ocorra. Distribuição beta e triangular são exemplos desses indicadores de estimativas do gerenciamento de riscos, onde o eixo (y), geralmente representa a probabilidade relativa, e o eixo $(\mathrm{X})$ pode representar, por exemplo o custo. 
Distribuição beta

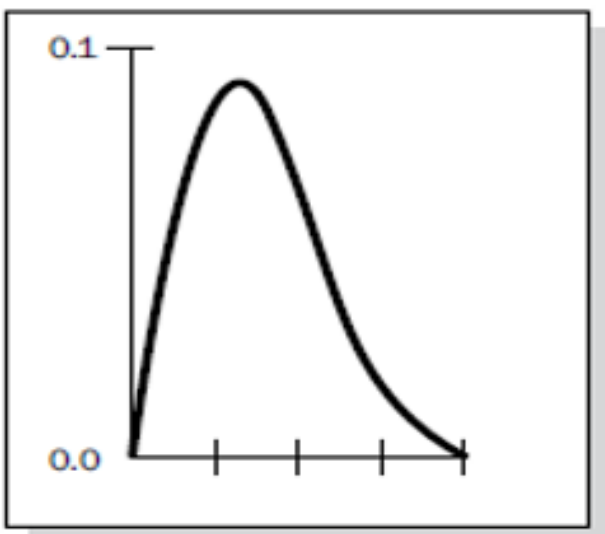

Distribuição triangular

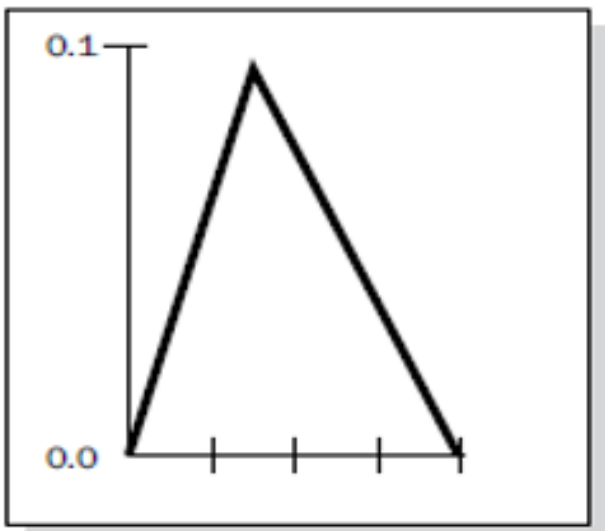

Figura 3 - Gráfico de distribuição beta e triangulas, são exemplos distribuições de probabilidade usada. Fonte: (PMBOK, 2013, p. 336).

Outro Indicador de suma importância da quantificação dos riscos é o Diagrama de Tornado. Esse indicador está ligado à análise da sensibilidade, é utilizado para determinar como a variação de um fator, uma variável impacta o objetivo do projeto. O conceito do caos se confunde muito nessa análise, pois conforme melhor essa analise é aplicada, mais transparência nas incertezas são evidenciadas, reduzindo a chance de eventos caóticos no futuro. (PMBOK, 2013) 


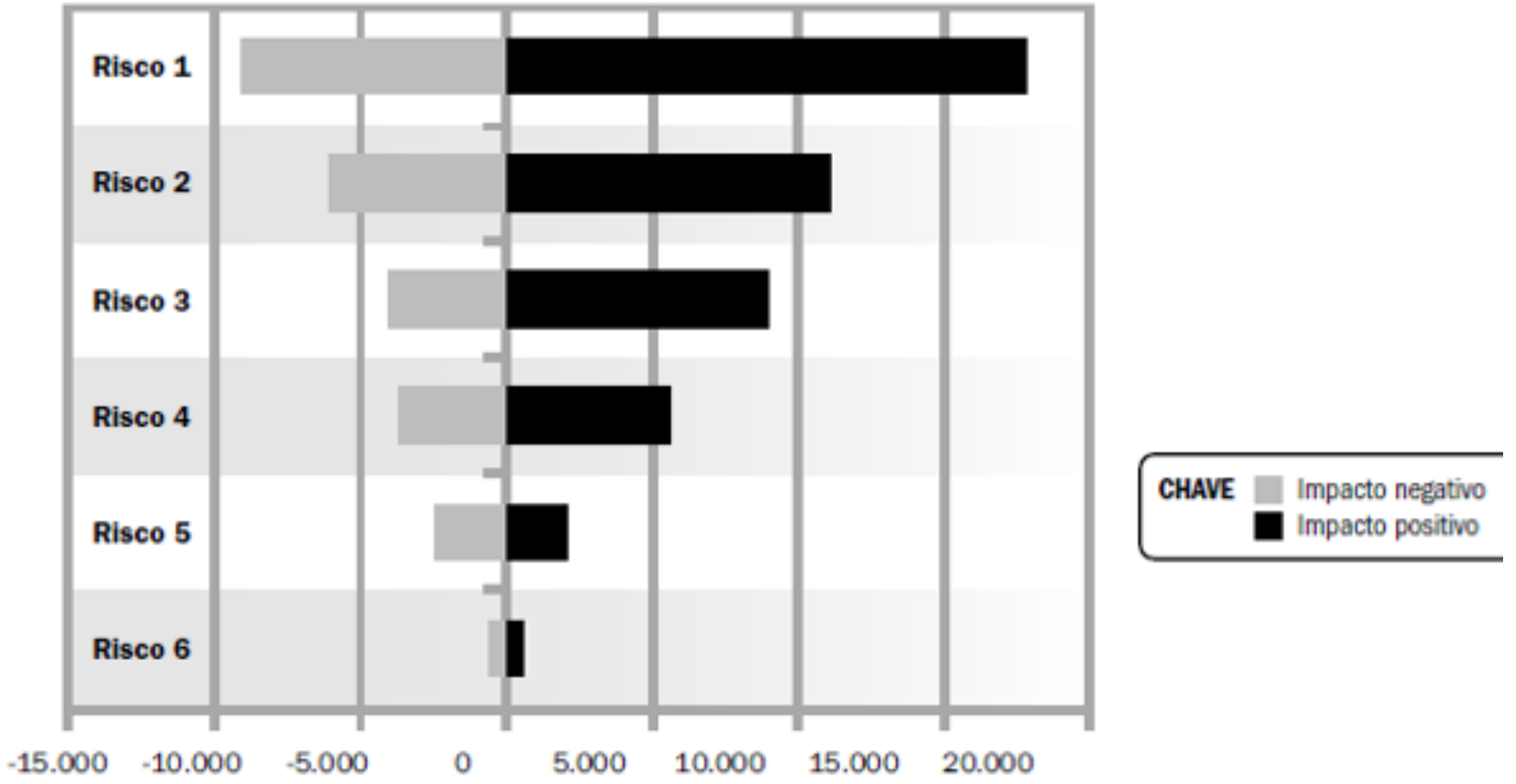

Figura 4 - Exemplo de Diagrama de Tornado. Fonte: (PMBOK, 2013, p.337)

\subsection{Planejar as respostas aos Riscos}

Este processo consiste no desenvolvimento de ações e opções para responder a oportunidade de ameaças dos riscos do projeto com alta probabilidade de ocorrência. Como já foi mencionando, os riscos podem ser gerenciados, mitigados, aceitos, transferidos ou prevenidos. Cabe a equipe ou o EGP (Escritório de Gerenciamento de Projetos) responsável pelo projeto, avaliar o Plano de gerenciamento do Projeto como um todo, e caso necessário, modifica-lo, dependo de como serão aplicados às ações de respostas aos riscos. As reservais gerencias e de contingencia deverão ser avaliadas, se os recursos aplicados e atividades programadas sobrepuserem essas reservas, os custo e cronogramas do projeto, deverão ser reavaliados. As respostas devem ser adequadas ao grau de risco a ser tratado, opiniões especializadas devem ser solicitas e métodos como o Delphi, que é uma técnica que reuni diversos questionamentos para organizar opiniões e respostas de um grupo de especialistas no assunto em questão, ADAMS (1982), e Brainstorm, estes devem ser incorporados neste processo para ajudar nas decisões a serem tomadas e gerenciadas em relação aos riscos. (PMBOK, 2013)

\subsection{Controlar os riscos}

Controlar os riscos é o processo de implementação de planos de respostas aos riscos, monitoramento dos riscos identificados, identificação de potenciais novos riscos, monitoramento dos resquícios dos riscos. Este processo, também são avaliados os desempenhos, do gerenciamento dos riscos durante todo o projeto, a fim de criar um histórico de tomadas de decisões para usar durante todo o ciclo de vida do projeto, ao passo que os riscos andam em paralelo a cada nova etapa, o risco de algum evento caótico pode ser premeditado, caso esses levantamentos tenham sido feitos de forma eficaz. Com isso, após 
experiência e maturidade adquirida nos processos anteriores, as respostas aos riscos são otimizadas para garantir o avanço do projeto. Ao fim de cada processo, são geradas saídas, documentos são atualizados, o plano de gerenciamento do projeto é aperfeiçoado, no caso do gerenciamento de riscos ser tão caótico em suas etapas, novos métodos podem ser empregados, de cardo com a modelagem de cada projeto, ou de acordo com a capacidade de ocorrência de eventos caóticos mudarem oque foi planejado, podendo ser estabelecido um novo modelo de gestão de riscos, especifico para o progresso do projeto em questão. (PMBOK, 2013).

\section{Conclusão}

Com a elaboração do deste artigo, percebe-se que a teoria do caos aplicado ao gerenciamento de riscos, ou qualquer aplicação organizacional de gestão pode quebrar paradigmas sobre métodos de gerenciamento, modelagem e simulação, pois todos os eventos relacionados a projeções e planejamentos são dinâmicos e estão expostos ao caos, podendo não ser tão previsíveis e gerenciáveis como descritos na maioria das literaturas do assunto.

Entendemos que no âmbito do gerenciamento de riscos de um projeto, as pessoas e sistemas estão trabalhando no limite e na eminencia de algum evento caótico. Os diversos fatores que podem contribuir para que o percentual das incertezas estipuladas durante as fases de planejamento, aumentem ao decorrer da implementação de ações, como: pessoas, fatores financeiros, eventos naturais, políticos, administrativos, entre outros. Independendo do fator preponderante, é importante que o gestor responsável pelo projeto, esteja preparado para visualizar todo ambiente dinâmicos interno e externo que esteja sensitivo a eventos caóticos as fases do projeto, para poder estabelecer estratégicas de contorno dessas situações infelizes que aconteceram. Não é pessimismo, mas deve-se gerenciar pensando que os riscos sempre podem se tonar o caos.

Vivemos em um mundo de transição turbulento, se o mesmo está mudando, novos estudos devem ser aprimorados para se adaptarem a essas novas transições. A teoria do caos, seus conceitos e aplicabilidades fazem parte dessa transformação, se entendermos o caos, nossa capacidade de superar as adversidades nos problemas encontrados aumentará, se entendermos que nem todo sistema, funciona de forma linear e previsível, nos tornaremos mais críticos. Poderemos atuar nas áreas influenciadas pelo caos de forma mais racional e inteligente.

\section{Referências}

ADAMS, John, et al Managing by Project Management. New York: univ Tech, 1983, 168 p.

BARALDINE, Paulo. Gerenciamento de Riscos. $3^{\text {a }}$ ed. Rio de Janeiro: Campus , 2010.

GIL, A.C. Como elaborar projetos de pesquisa. 4 ed. - São Paulo: Altas, 2002.

HUBBARD, D.W. "How to measure anything: finding the value of intagibles in business". John Wiley \& Sons, Haboken, 2007.

KERZNER, Harold. Gestão de projetos: as melhores práticas. New York: John Willey \& Sons, 2009. 
LORENZ, E. N. A Essência do Caos. Brasília. UnB, 1996.

PMI. Um guia de conhecimento em gerenciamento de projetos. Guia PMBOK $5^{\mathrm{a}}$. Ed - EUA Project Management Institute, 2013

PRIGOGINE, I. As leis do caos. Tradução de Roberto Leal Ferreira. São Paulo: Editora Unesp, 2002.

RODRIGUES Eli. 21 Erros Clássicos da Gestão de Projetos. Rio de Janeiro: Brasport, 2014.

VIEIRA, E. N.O. Gerenciando Projetos na Era de Grandes Mudanças. Uma Breve Abordagem do Panorama Atual. $\quad$ Anais eletrônicos... em:<http://www.pmisp.org.br/exe/artigos/eduardonewton_artigogprojetosi_pdf $>$ Acesso em: 10 de ago.2013.

[1] Engenharia de Produção, com ênfase em Gestão da Qualidade e Ferramentas, Avaliação da Conformidade e gerenciamento de riscos com ênfase me GED.

\section{PUBLIQUE SEU ARTIGO CIENTÍFICO EM:}

https://www.nucleodoconhecimento.com.br/enviar-artigo-cientifico-para-submissao 